\title{
First female Korean child with Coffin-Lowry synd- rome: a novel variant in RPS6KA3 diagnosed by exome sequencing and a literature review
}

\author{
Ari Song \\ Minji Im², \\ Min-Sun Kim ${ }^{3}$, \\ Eu Seon $\mathrm{Noh}^{3}$, \\ Chiwoo $\mathrm{Kim}^{3}$, \\ Jahyun Jang ${ }^{4}$, \\ Sae-Mi Lee, ${ }^{5,6}$, \\ Chang-Seok Ki ${ }^{5}$ \\ Sung Yoon $\mathrm{Cho}^{3}$, \\ Dong-Kyu Jin ${ }^{3}$
}

'Department of Pediatrics, Incheon Sejong Hospital, Incheon, Korea

${ }^{2}$ Department of Pediatrics, Sungae Hospital, Seoul, Korea

${ }^{3}$ Department of Pediatrics, Samsung Medical Center, Sungkyunkwan University School of Medicine, Seoul, Korea

${ }^{4}$ Department of Laboratory Medicine and Genetics, Samsung Medical Center Sungkyunkwan University School of Medicine, Seoul, Korea

${ }^{5}$ Green Cross Genome, Yongin, Korea ${ }^{6}$ Department of Laboratory Medicine, Kangwon National University School of Medicine, Chuncheon, Korea

Received: 28 June, 2021

Revised: 31 July, 2021

Accepted: 6 August, 2021

Address for correspondence:

Sung Yoon Cho

Department of Pediatrics, Samsung Medical Center, Sungkyunkwan University School of Medicine, 81 Irwon-ro, Gangnam-gu, Seoul 06351, Korea

Email: nadri1217@naver.com

https://orcid.org/0000-0003-2913059X
Coffin-Lowry syndrome (CLS, OMIM \# 303600) is a rare X-linked disorder caused by mutations in RPS6KA3. CLS is characterized by facial dysmorphism, digit abnormalities, developmental delays, growth retardation, and progressive skeletal changes in male patients. Females with CLS are variably affected, complicating diagnosis. Here, we describe the clinical and molecular findings in a female Korean child with CLS and review the associated literature. A 5-year-old girl presented with short stature and developmental delays. She had a coarse facial appearance characterized by a prominent forehead, hypertelorism, thick lips, and hypodontia. She also had puffy tapering fingers and pectus excavatum. We performed exome sequencing and identified a novel, likely pathogenic, heterozygous variant, c.326_338delinsCTCGAGAC (p.Val109Alafs*10), in RPS6KA3 (NM_004586.2). This is the first Korean female genetically diagnosed with CLS. In contrast to the delayed bone age reported in previous studies, our patient showed advanced bone age and central precocious puberty. CLS should be considered as a differential diagnosis of short stature, tapering fingers, and developmental delay. We suggest that molecular techniques can be a useful tool for diagnosis of rare disorders such as CLS because such conditions are not simple, and the associated spectrum of phenotypes can vary.

Keywords: Coffin-Lowry syndrome, RPS6KA3, X-linked inheritance, Short stature, Developmental delay

\section{Highlights}

Coffin-Lowry Syndrome (CLS) is a rare X-linked disorder caused by mutations in RPS6KA3. We describe the clinical and molecular analysis of a five-year-old girl with CLS who presented with short stature, facial dysmorphism, tapering fingers, and developmental delays. This is the first Korean female identified with CLS and the first case of central precocious puberty (CPP) in CLS.

\section{Introduction}

Coffin-Lowry syndrome (CLS, OMIM \# 303600) is an X-linked inherited disorder caused by loss-of-function mutations in the Ribosomal Protein S6 Kinase Polypeptide 3 (RPS6KA3) gene located in Xp22.2. ${ }^{1)}$ CLS is a very rare disease, with an estimated incidence that varies from 1:40,000 to $1: 100,000 .{ }^{1,2)}$ Approximately $70 \%-80 \%$ of cases are sporadic, whereas $20 \%-30 \%$ have more than one additional affected family member. ${ }^{1,2}$ Affected individuals display a wide range of phenotypes, including short stature, facial dysmorphism, tapering fingers, hearing deficits, intellectual disabilities, developmental delays, and progressive skeletal 


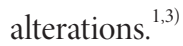

A CLS diagnosis in males can be established using clinical manifestations, including intellectual disabilities, characteristic facial and skeletal findings, and radiographic findings. ${ }^{4}$ However, females with CLS show various clinical features, ranging from tapering fingers with normal facial appearance and intelligence to remarkable facial dysmorphism with moderate intellectual disability. ${ }^{1,2)}$ These various clinical manifestations hinder CLS diagnosis in females compared to males. Also, establishing a CLS diagnosis in very young children can be much more difficult than in older patients because the physical characteristics are milder and not specific. ${ }^{2,4)}$

Here, we describe clinical and molecular analysis of a 5-yearold girl with CLS who presented with short stature, facial dysmorphism, tapering fingers, and developmental delays. We identified a novel, likely pathogenic, heterozygous variant, c.326_338delinsCTCGAGAC (p.Val109Alafs ${ }^{\star} 10$ ), in RPS6KA3 by exome sequencing. This is the first Korean female identified with CLS and the first case of central precocious puberty (CPP) in CLS. We also review the literature related to females with CLS.

\section{Case report}

A 5-year-old girl presenting with short stature and developmental delays visited Samsung Medical Center. This child was born at 40 weeks gestational age by vaginal delivery and had no history of perinatal problems. Her birth weight was 3,210 $\mathrm{g}$ (25th-50th percentile). The patient was the only child of nonconsanguineous healthy Korean parents. The father's height was $182 \mathrm{~cm}$, and the mother's height was $168 \mathrm{~cm}$. A newborn screening test for inherited metabolic disorders returned normal results. She presented with hypotonia and hyperlaxity of the joints at the age of 1 month.

At the age of 5 years 5 months, the child's height was $106 \mathrm{~cm}$ (standard deviation score [SDS], -1.386), her weight was 20.0

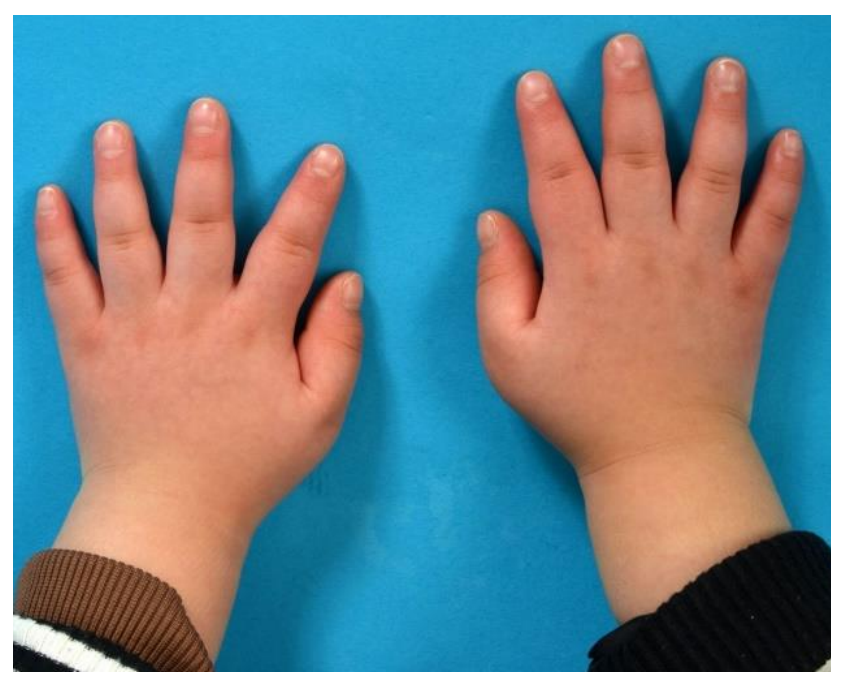

Fig. 1. Puffy hands with typical tapering stubby fingers were seen in a girl with Coffin-Lowry syndrome at age 5 years 5 months. $\mathrm{kg}$ (SDS, 0.104), and her head circumference was $50.0 \mathrm{~cm}$ (SDS, -0.357). SDS was estimated using the 2017 Korean National Growth Chart. ${ }^{5}$ She showed facial dysmorphism, including a prominent forehead, hypertelorism, downward-slanting palpebral fissures, thick lips, and micrognathia. In addition, pectus excavatum and tapering stubby fingers were noted (Fig. $1)$.

She started to walk at 18 months of age and used 2-word sentences from the age of 5 years. At the time of her visit to our clinic, she was able to run independently and ascend stairs, but her gait was unstable, and she fell frequently. Her receptive language development was preserved, but expressive language development was delayed by about 18 months, and her pronunciation was inaccurate. The K-DST (Korean Developmental Screening Test for Infants and Children) at 65 months of age revealed global developmental delays that required further evaluation in all areas (gross motor, 4 ; fine motor, 3; cognition, 6; language, 8; sociality, 8; and self-control, 8).

The laboratory test results (complete blood count, electrolytes, glucose, blood gases, hepatic and renal function tests, and urinalysis) were normal. Other endocrine evaluations were also normal: triiodothyronine, $111.62 \mathrm{ng} / \mathrm{dL}(60-181$ $\mathrm{ng} / \mathrm{dL})$; thyroid-stimulating hormone, $2.431 \mu \mathrm{IU} / \mathrm{mL}(0.64-$ $69.27 \mu \mathrm{IU} / \mathrm{mL})$; free thyroxine, $1.33 \mathrm{ng} / \mathrm{dL}(0.89-1.8 \mathrm{ng} / \mathrm{dL})$; 25-hydroxyvitamin D3, $17.9 \mathrm{ng} / \mathrm{mL}(10-150 \mathrm{ng} / \mathrm{mL})$; insulinlike growth factor-1 (IGF-1), $174.5 \mathrm{ng} / \mathrm{mL}(70.2-300.3 \mathrm{ng} /$ $\mathrm{mL}$ ); and alkaline phosphatase, $282 \mathrm{IU} / \mathrm{L}(142-335 \mathrm{IU} / \mathrm{L})$. Chromosome analysis revealed 46, XX. Bone age at 5 years 7 months was 2 months older than chronologic age. Lumbar scoliosis was noted on a skeletal survey.

Echocardiography and electroencephalogram (EEG) results were normal. A brain magnetic resonance imaging scan revealed multiple white matter lesions, mainly on the bilateral frontal lobe with a left paraventricular cyst. Dental examination revealed malocclusion and hypodontia (congenitally missing first molars). Ophthalmological examination showed astigmatism. The patient's hearing was normal.

To make a diagnosis and identify underlying genetic defects, we listed the causative genes of candidate diseases based on several key symptoms (Supplementary Table 1) from the medical history and laboratory and radiographic findings. We suspected RASopathies, including Noonan syndrome and Costello syndrome. When the patient was 5 years 5 months old, exome sequencing was performed to screen for pathogenic variants of genes responsible for diseases resulting in short stature, facial dysmorphism, and developmental delays. Genomic DNA was extracted from the patient's peripheral blood. Approximately 57,000 target exons of a total of 4,503 genes were captured through the $x G e n{ }^{\circledR}$ Inherited Diseases Panel (Integrated DNA Technologies, Inc., Coralville, IA, USA) and sequenced with an Illumina NextSeq 500 (Illumina Inc., San Diego, CA, USA) for $2 \times 150$ bp paired-end reads. Sequence reads were mapped to the hg19 human reference sequence using the Burrow-Wheeler Aligner version 0.7.12. The average panel depth was 166X, and 
99.7\% of bases were above 10X. No pathogenic variant was detected in the ATRX, HRAS, MAP2K1, PHF6, or PTPN11 gene, which are associated with RASopathies. A novel, likely pathogenic, heterozygous variant, c.326_338delinsCTCGAGAC (p.Val109Alafs ${ }^{\star} 10$ ), was identified in RPS6KA3 (Fig. 2). This variant had not been previously reported and was not found in her parents or the control databases: 1,000 genomes (http:// phase3browser.1000genomes.org), Exome Variant Server (http://evs.gs.washington.edu/EVS/), and gnomAD (https:// gnomad.broadinstitute.org/). The novel heterozygous variant, c.326_338delinsCTCGAGAC (p.Val109Alafs ${ }^{\star} 10$ ), is a truncated variation and is likely pathogenic according to the American College of Medical Genetics and Genomics Standards and Guidelines. ${ }^{6}$ Eventually, the patient was diagnosed with CLS.

At the age of 7 years, the patient experienced her first sudden fall to the ground after an unexpected sound stimulus. Since that time, the frequency of fall episodes triggered by sudden sound stimuli has increased to dozens per day. Stimulus-induced drop episodes (SIDEs) were diagnosed using simultaneous surface electromyogram (sEMG) and video EEG results.

At 7 years nine months, the patient's breast development was Tanner stage II, and her bone age had advanced to 9 years 6 months. Her height was $122.7 \mathrm{~cm}$ (SDS, -0.541), and her weight was $25.0 \mathrm{~kg}$ (SDS, -0.237). The Bayley-Pinneau method was applied to predict an adult height of $151.8 \mathrm{~cm}$, which is shorter than the midparental height of $163.5 \mathrm{~cm}$. Laboratory tests yielded the following results: estradiol $1 \mathrm{pg} / \mathrm{mL}(<20 \mathrm{pg} / \mathrm{mL})$, luteinizing hormone (LH) $1.1 \mathrm{mIU} / \mathrm{mL}(<0.02-0.3 \mathrm{mIU} / \mathrm{mL})$, follicle stimulating hormone $1.6 \mathrm{mIU} / \mathrm{mL}(0.5-6.0 \mathrm{mIU} / \mathrm{mL})$, and IGF-1 $273.2 \mathrm{ng} / \mathrm{mL}(102.3-460.1 \mathrm{ng} / \mathrm{mL})$. The peak $\mathrm{LH}$ level was $5.7 \mathrm{mIU} / \mathrm{mL}$ on a gonadotropin-releasing hormone $(\mathrm{GnRH})$ stimulation test. GnRH agonist therapy was initiated to treat CPP.

At 8 years seven months, the patient's scoliosis was $23^{\circ}$ of the Cobb angle (Fig. 3). She was able to communicate with others, write her name, and count to eight. She was able to walk independently but fell approximately 10 times a day due to hypotonia. Clobazam was prescribed to control SIDEs, but we changed that to clonazepam due to resulting drowsiness. SIDE frequency was reduced to $30 \%$ with clonazepam. Leuprolide $3.75 \mathrm{mg}$ was administered once every four weeks, and pubertal development regressed.

\section{Ethical statement}

This study was approved by the Institutional Review Board of Samsung Medical Center (approval number: 2012-05-080-008). Informed consent was obtained from her parents.

\section{Discussion}

Descriptions of the clinical features of CLS in females are

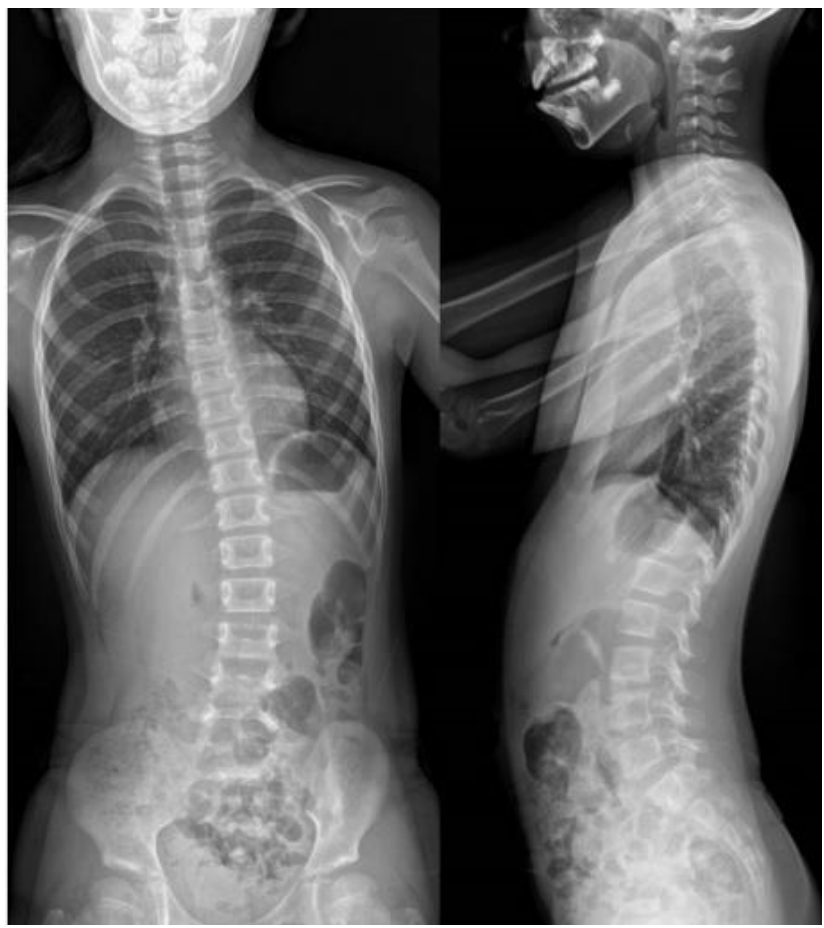

Fig. 3. Anterioposterior and lateral radiographs of the spine showing scoliosis with a $23^{\circ} \mathrm{Cobb}$ angle.

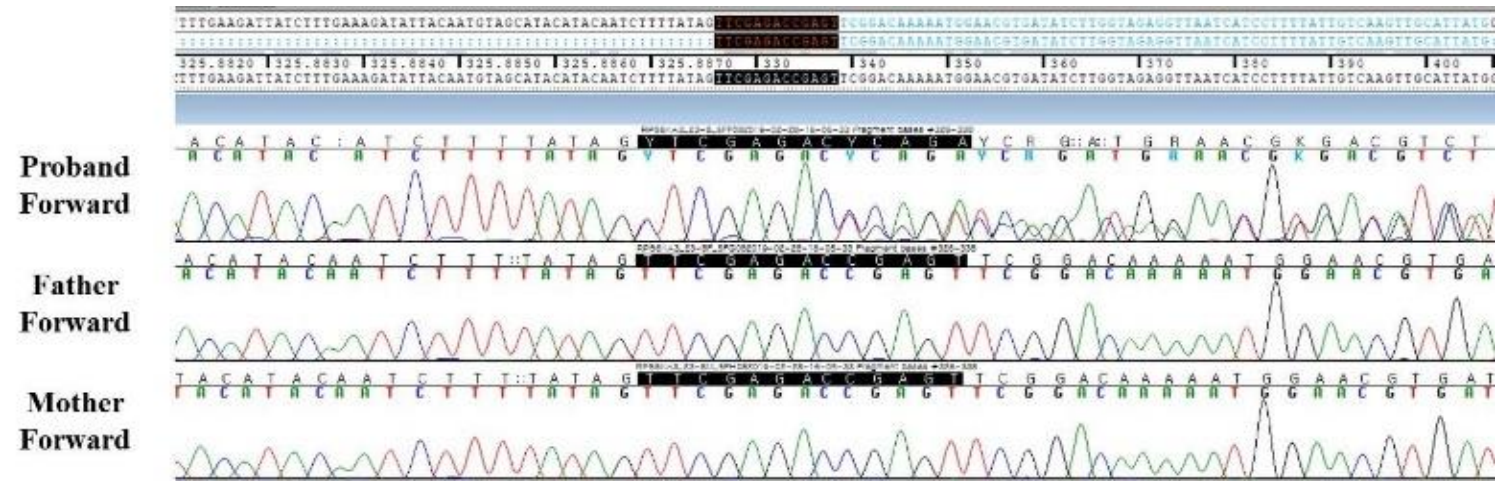

Fig. 2. Sanger sequencing of the proband and her parents. A heterozygous, likely pathogenic variant, c.326_338delinsCTCGAGAC (p.Val109Alafs*10), in RPS6KA3 was found in the patient but was absent in her parents. 


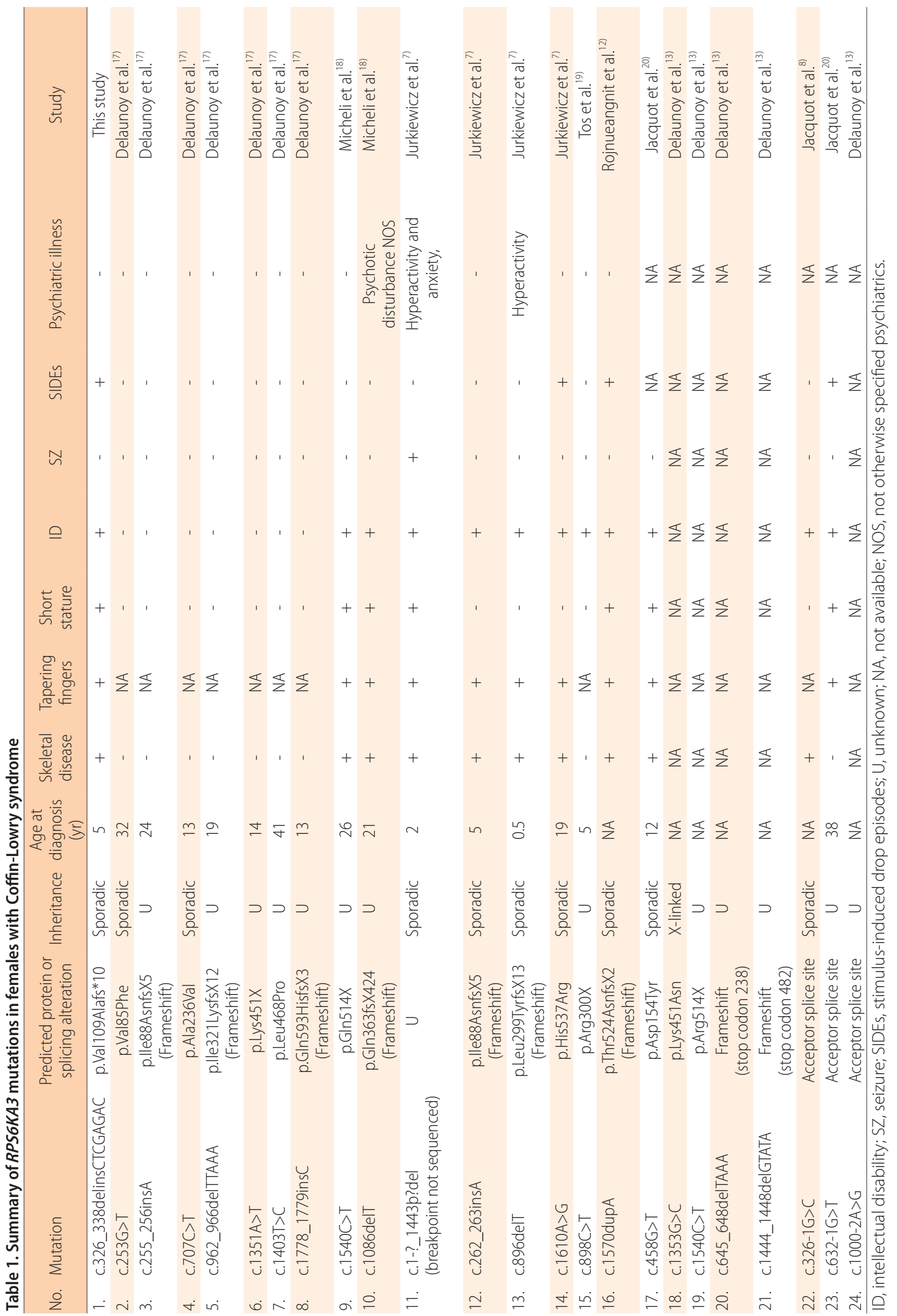


rare in the literature. Here, we presented the clinical features and natural course of a female CLS patient. CLS diagnosis in very young children is often difficult because the physical characteristics are mild and non-specific. ${ }^{1,4)}$ Moreover, female heterozygotes can show variable involvement. ${ }^{3,7)}$ CLS can be confused with other syndromes, including ATRX (alphathalassemia-X-linked intellectual disability) syndrome, Borjeson-Forssman-Lehmann syndrome, and Williams syndrome, which also are characterized by short stature, facial dysmorphism, and developmental delays. ${ }^{2,4)}$ Those diseases can be difficult to differentiate from CLS clinically, but a genetic evaluation can facilitate differentiation among them.

RPS6KA3 is located at Xp22.2, encompasses 22 exons, and encodes the S6K alpha 3 monomer protein that acts downstream of the mitogen-induced Ras-Activated Protein Kinase cascades. These have an important role in cell-cycle progression, differentiation, and cell survival. ${ }^{8)}$ RPS6KA3 expression activates the cAMP response element-binding protein, which plays an important role in human learning and memory. The cellular capacity to activate RPS6KA3 is key for human cognitive performance.

Furthermore, studies using a fibroblast cell line derived from a CLS patient indicate that the cells are defective in epidermal growth factor-induced c-fos gene expression, which regulates the differentiation and activity of specific bone cell populations. ${ }^{10)}$ The transcription factor ATF4 acts as a critical substrate of RPS6KA3, and lack of ATF4 phosphorylation can interrupt the regulatory role of ATF4 in osteoblast differentiation. Those features could explain the cognitive impairment and skeletal anomalies seen in CLS caused by RPS6KA mutations. ${ }^{11)}$

To date, more than 182 mutations have been identified in RPS6KA3 (HGMD Professional database, version 2020.4). The RPS6KA3 mutational spectrum includes 82 missense/ nonsense mutations, 28 splice site variants, 33 small deletions ( $\leq 20 \mathrm{bp}$ ), 15 small insertions ( $\leq 20 \mathrm{bp}$ ), 1 small indel ( $\leq 20 \mathrm{bp}$ ), 14 gross deletions, and nine gross insertions. The genotypephenotype correlation of CLS is unclear, and clinical features differ among patients from one family. CLS is inherited in an $\mathrm{X}$-linked dominant pattern. Various findings on the skewing of X-inactivation have been demonstrated in previous studies of female CLS patients, but no associations have been found between the X-inactivation pattern and the presentation or severity of CLS manifestations. ${ }^{7,12)}$ As shown in Table 1, female heterozygotes have a wide range of observed clinical features. ${ }^{4,12,13)}$ Our patient was diagnosed with CLS at the age of 5 , which is much earlier than is typical of female CLS patients (Table 1). Hyperextensible joints, tapering fingers, and hypotonia can be present at birth. These features were also observed in our patient. Early CLS diagnosis enables early detection and treatment of skeletal manifestations, such as spinal kyphosis/ scoliosis. Intervention and rehabilitation in the early stages of developmental delay support the patient's clinical progress. Unlike males with CLS, affected females can have psychological problems. Because these symptoms progress with age, follow-up in young female patients is necessary. ${ }^{2,3}$
SIDEs have been reported in 13\%-20\% of males and 3\%-7\% of females with CLS. ${ }^{12,14)}$ These episodes are characterized by a paroxysmal drop attack without loss of consciousness induced by unexpected auditory or tactile stimuli. ${ }^{2}$ The pathophysiology of SIDEs is not well understood. Several studies have shown that epileptiform activity is absent on EEG during SIDEs. A sudden transient decrease in sEMG activity is usually observed during SIDEs, but one previous report showed increased sEMG activity. ${ }^{14)}$ We found SIDEs in hyperekplexia with increased sEMG activity in our patient.

Two previous reports of CLS have been made in Korea, 1 case in a 12-month-old male who was diagnosed clinically and 1 case in an 18-year-old male who was diagnosed by a mutation, c.889_890delAG (p.Leu298Phefs ${ }^{\star} 21$ ), in RPS6KA3. ${ }^{15,16)}$ The patient's mother in the second case was a carrier of the same mutation, but she had no clinical signs or symptoms of CLS. ${ }^{16)}$

CLS is associated with short stature, delayed bone age, and delayed puberty. ${ }^{1,2)}$ This study differed from previous reports in that our patient showed advanced bone age, acceleration of bone maturation, and CPP. The cause of CPP in this patient is unclear and is considered spontaneous. To the best of our knowledge, no previous reports have linked CLS with CPP and treatment with a GnRH agonist, but it could be helpful in managing CLS in the future.

In conclusion, we diagnosed CLS in a young female child using next generation sequencing because diagnosis based on clinical symptoms is difficult. We report the first female Korean child with CLS and identified a novel, likely pathogenic, heterozygous variant in RPS6KA3. We suggest molecular techniques as a helpful tool in early diagnosis of rare diseases with a variable spectrum of phenotypes.

\section{Notes}

Supplementary material: Supplementary Table 1 can be found at https://doi.org/10.6065/apem.2142134.067.

Conflicts of interest: No potential conflict of interest relevant to this article was reported.

Funding: This study received no specific grant from any funding agency in the public, commercial, or not-for-profit sectors.

Author contribution: Conceptualization: SYC, DKJ; Data curation: AS, MSK, ESN, CK, SYC; Methodology: AS, MI, JJ, SML; Visualization: JJ, SML, CSK; Writing - original draft: AS; Writing - review \& editing: SYC, DKJ

\section{ORCID}

Ari Song: 0000-0003-3689-5312

Minji Im: 0000-0002-8114-844X

Min-Sun Kim: 0000-0002-7799-3229

Eu Seon Noh: 0000-0001-8004-0157

Chiwoo Kim: 0000-0003-1956-2380

Jahyun Jang: 0000-0003-0516-4947

Sae-Mi Lee: 0000-0002-0462-6700

Chang-Seok Ki: 0000-0001-7679-8731

Sung Yoon Cho: 0000-0003-2913-059X 
Dong-Kyu Jin: 0000-0003-4162-2706

\section{References}

1. Rogers RC, Abidi FE. Coffin-Lowry syndrome. In: Adam MP, Ardinger HH, Pagon RA, Wallace SE, Bean LJH, Stephens K, Amemiya A, editors. GeneReviews" [Internet]. Seattle (WA): University of Washington,Seattle; 1993-2020 [updated 2018 Feb 1; cited 2002 Jul 16]. Available from http://www.ncbi.nlm.nih.gov/books/NBK1346/.

2. Pereira PM, Schneider A, Pannetier S, Heron D, Hanauer A. Coffin-Lowry syndrome. Eur J Hum Genet 2010;18:62733.

3. Hanauer A, Young ID. Coffin-Lowry syndrome: clinical and molecular features. J Med Genet 2002;39:705-13.

4. Touraine R-L, Zeniou M, Hanauer A. A syndromic form of X-linked mental retardation: the Coffin-Lowry syndrome. Eur J Pediatr 2002;161:179-87.

5. Kim JH, Yun S, Hwang SS, Shim JO, Chae HW, Lee YJ, et al. The 2017 Korean National Growth Charts for children and adolescents: development, improvement, and prospects. Korean J Pediatr 2018;61:135-49.

6. Richards S, Aziz N, Bale S, Bick D, Das S, Gastier-Foster J, et al. Standards and guidelines for the interpretation of sequence variants: a joint consensus recommendation of the American College of Medical Genetics and Genomics and the Association for Molecular Pathology. Genet Med 2015;17:405-24.

7. Jurkiewicz D, Jezela-Stanek A, Ciara E, PiekutowskaAbramczuk D, Kugaudo M, Gajdulewicz M, et al. Four novel RSK2 mutations in females with Coffin-Lowry syndrome. Eur J Med Genet 2010;53:268-73.

8. Jacquot S, Merienne K, Pannetier S, Blumenfeld S, Schinzel A, Hanauer A. Germline mosaicism in Coffin-Lowry syndrome. Eur J Hum Genet 1998;6:578-82.

9. Harum KH, Alemi L, Johnston MV. Cognitive impairment in Coffin-Lowry syndrome correlates with reduced RSK2 activation. Neurology 2001;56:207-14.
10. De Cesare D, Jacquot S, Hanauer A, Sassone-Corsi P. Rsk2 activity is necessary for epidermal growth factor-induced phosphorylation of CREB protein and transcription of c-fos gene. Proc Natl Acad Sci U S A 1998;95:12202-7.

11. Yang X, Matsuda K, Bialek P, Jacquot S, Masuoka HC, Schinke T, et al. ATF4 is a substrate of RSK2 and an essential regulator of osteoblast biology; implication for CoffinLowry Syndrome. Cell 2004;117:387-98.

12. Rojnueangnit K, Jones JR, Basehore MJ, Robin NH. Classic phenotype of Coffin-Lowry syndrome in a female with stimulus-induced drop episodes and a genotype with preserved N-terminal kinase domain. Am J Med Genet A 2014;164A:516-21.

13. Delaunoy JP, Abidi F, Zeniou M, Jacquot S, Merienne K, Pannetier S, et al. Mutations in the X-linked RSK2 gene (RPS6KA3) in patients with Coffin-Lowry syndrome. Hum Mutat 2001;17:103-16.

14. Nelson GB, Hahn JS. Stimulus-induced drop episodes in Coffin-Lowry syndrome. Pediatrics 2003;111:e197-202.

15. Shin JE, Seo ES, Lee DH. A case of Coffin-Lowry syndrome. J Genet Med 2007;4:196-9.

16. Yoon JY, Cheon CK. Coffin-Lowry syndrome - the first genetically confirmed case in Korea diagnosed by whole exome sequencing. J Int Gen 2020;2:10-2.

17. Delaunoy JP, Dubos A, Marques Pereira P, Hanauer A. Identification of novel mutations in the RSK2 gene (RPS6KA3) in patients with Coffin-Lowry syndrome. Clin Genet 2006;70:161-6.

18. Micheli V, Sestini S, Parri V, Fichera M, Romano C, Ariani F, et al. RSK2 enzymatic assay as a second level diagnostic tool in Coffin-Lowry syndrome. Clin Chim Acta 2007;384:3540.

19. Tos T, Alp MY, Aksoy A, Ceylaner S, Hanauer A. A familial case of Coffin-Lowry syndrome caused by RPS6KA3 C.898C $>$ T mutation associated with multiple abnormal brain imaging findings. Genet Couns 2015;26:47-52.

20. Jacquot S, Merienne K, De Cesare D, Pannetier S, Mandel JL, Sassone-Corsi P, et al. Mutation analysis of the RSK2 gene in Coffin-Lowry patients: extensive allelic heterogeneity and a high rate of de novo mutations. Am J Hum Genet 1998;63:1631-40. 